\title{
Modification of the Immune Response in Rats by Di-octyl Phthalate
}

\author{
Key words : Di-octyl phthalate-Immunomodulation-Rat
}

Ever since the first symposium on phthalate held during 1973 in U.S.A., many reviews have been published on biological effects of phthalic acid esters. ${ }^{1}$ These reports, however, have shed no insight into the likely adverse effects of cumulative doses of phthalate on immune system, which is one of many potential targets of foreign chemicals. The specific lesions of the immune system may lead to the damage of organs and eventually compromise the general well being of the individual. Our recent subchronic toxicity study with di-octyl phthalate (DOP) indicated sensitivity of lymphoid organs and the partial evidence of alteration in immunity in rats. ${ }^{2)}$ This prompted us to specifically investigate the effect of short term exposure to DOP in rats with special reference to immune system and on host resistance to endotoxin sensitivity and parasitic challenge-Nippostrongylus brasiliensis—rat model.

Two-month-old male albino rats obtained from ITRC animal colony were used and provided with pellet diet (Hind Lever, India) and water ad libitum. Di-octyl phthalate (DOP: $\left.\mathrm{C}_{6} \mathrm{H}_{4}-1,2\left(\mathrm{COO}\left(\mathrm{CH}_{2}\right)_{7} \mathrm{CH}_{3}\right)_{2} ; \mathrm{MW} 390.57\right)$ an isomer of Di-2-ethylhexyl phthalate (DEHP) was obtained from Ranbaxy Laboratories Ltd. (India) and its acute oral $\mathrm{LD}_{50}$ value in male rat worked out as $53.7 \mathrm{~g} / \mathrm{kg}$ body weight.

One hundred eight rats were randomly divided into 4 equal groups. The first three groups were treated orally with $0.05,0.1$ or $0.2 \times \mathrm{LD}_{50}$ DOP for 5 days, respectively, while the 4 th group received only $0.5 \mathrm{ml}$ physiologic saline per animal and served as control. Fortyeight hours after the last dose 6 animals from each group were sacrificed, autopsy performed and various organs examined histologically.

Out of the remaining 84 rats pretreated with DOP for 5 days, 20 rats (5 rats/group) were injected i.p. with SRBC $48 \mathrm{hrs}$ after the last dose of DOP for assessing the alteration in humoral immunity using plaque-forming cell (PFC) assay for detecting antibodies of IgM class. ${ }^{3}$ Additionally the anti-SRBC antibody titer was also determined in the serum and the data are expressed as $\log _{2}$ titer. For evaluating alterations in indirect immunity 40 rats pretreated with DOP for 5 days (10 rats/group) were used. Fortyeight hours after last dose the animals were subjected to harvesting of peritoneal exudate cells to be used for a) the assessment of nitroblue tetrazolium reduction expressed as optical density measured at $515 \mathrm{~nm},{ }^{4)}$ and b) their ability to phagocytose opsonized SRBC and expressed as per cent phagocytosis. ${ }^{5)}$ Remaining 24 rats (6 rats/group) pretreated 
with DOP for 5 days were infected $48 \mathrm{hrs}$ posttreatment by s.c. inoculation of of $1000 \mathrm{~L}_{3}$ larvae of $N$. brasiliensis per animal. Ten days later rats in all groups were killed by etherization for total worm counts in the intestine excluding the distal large intestine. $\left.{ }^{6}\right)$

For additional studies of bacterial endotoxin sensitivity 40 rats were randomly divided into 2 equal groups of control and experimental $\left(0.2 \times \mathrm{LD}_{50} \mathrm{DOP}\right.$ for 5 days) for the treatment. Fortyeight hours after the last dose each rat then received an i.v. injection of endotoxin ( $E$. coli lipopolysaccharide 0127:B8 Difco, USA) at any of the following doses: $60,125,250$ or $500 \mu \mathrm{g} / \mathrm{rat}$ and mortality scored $48 \mathrm{hrs}$ postinjection in all the groups. ${ }^{7)}$ All the data were analysed using student's ' $\mathrm{t}$ ' test.

Oral administration of DOP caused dose dependent histological evidence of depletion of cellular population in the periarteriolar lymphoid sheet (PALS) in spleen but without any evidence of gross toxicity. Thymus also exhibited dose dependent changes in cellularity resulting in loss of distinction between cortex and medulla. There was no evidence of degeneration or necrosis in thymic cells. The regional and peripheral lymph nodes revealed moderate to marked loss in the activity of germinal centers only. Other organs, however, did not show any specific histologic change attributable to DOP treatment.

DOP exposure caused a dose dependent reduction in the antibody synthesis on 4th day following SRBC immunization (primary immune response) resulting in several folds lowering $(\mathrm{P}<0.001)$ in the number of $\operatorname{IgM}$ producing spleen cells (Fig. 1A). Similarly there was concomitant dose related significant reduction

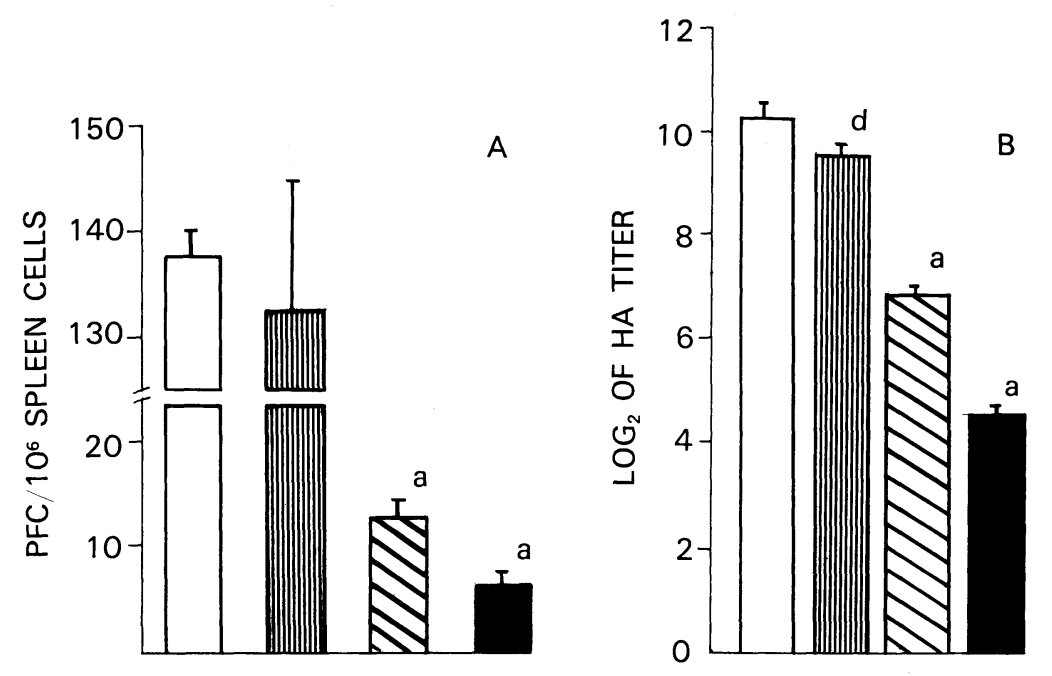

Fig. 1. (A) Plaque forming cells in spleen, (B) Antibody in serum : ( $\square$ )

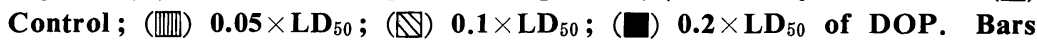
marked with (a) indicate $p<0.001$; (d) $p<0.05$ compared to control group. 

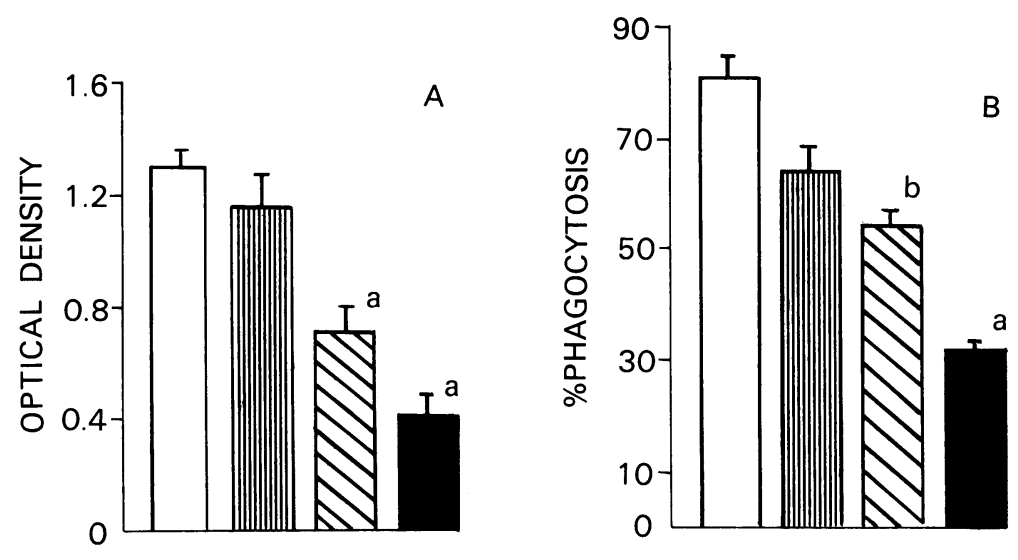

Fig. 2. Reduction of nitroblue tetrazolium by peritoneal exudate cells expressed as O.D., (B) Phagocytosis of SRBC by adherent peritoneal exudate

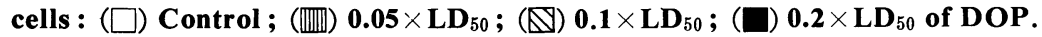
Bars marked with (a) indicate $p<0.0001$; (b) $p<0.01$ compared to control group.

in the serum anti-SRBC HA titer on the 4th day of immunization (Fig. 1B). Furthermore DOP treated rats showed a dose response change in the ability of peritoneal exudate cells (PECs) to reduce the nitroblue tetrazolium (NBT) where the highest or middle dose of DOP caused significant decrease in the optical density $(\mathrm{P}<0.001)$ indicating the impairment of metabolic functions (Fig. 2A). In addition, DOP treatment also affected considerably the phagocytic potential of adherent PECs for opsonized SRBC (Fig. 2B). Highest dose of DOP produced $60 \%$ reduction $(\mathrm{P}<0.001)$ in phagocytosis.

Amongst host resistance assay of parasitic challenge of $N$. brasiliensis infection of rat a definite trend in the increased worm burden of $19 \%$ and $30 \%$ over the control was observed by day 10 in the DOP treated groups of $0.1 \times \mathrm{LD}_{50}$ and $0.2 \times \mathrm{LD}_{50}$ dose levels respectively. Additionally the results of bacterial endotoxin shock (LPS) especially with 125 and $250 \mu \mathrm{g}$ doses revealed 2 and 2.5 folds increase respectively in the mortality pattern of DOP treated rats over non-treated controls.

Our earlier studies demonstrated that in vitro exposure of splenocytes to very low doses of DOP caused some indication of functional impairment together with ultrastructural alterations. ${ }^{8)}$ The histopathological findings of the present report further confirmed the sensitivity of lymphoreticular tissues as early as at 7 days of DOP treatment. This was predominantly reflected when the DOP treated rats exhibited suppression of primary humoral immune response as evinced by significant reduction in PFCs and serum anti-SRBC antibody titer. On the basis of our current and past data it would seem clear that DOP is capable of inducing depression of B-lymphocyte responses. However, the 
possibility of interrelationship between decreased zinc level in phthalate-induced testicular injury9) and reduced antibody synthesis due to altered $\mathrm{T}$ helper function of atrophic thymus as a result of zinc deficiency ${ }^{10)}$ remains to be ascertained.

Further the impairment observed in the reducing ability of nitroblue tetrazolium by PECs together with diminished phagocytosis of SRBC by adherent PECs from DOP treated rats were suggestive of another likely target site of DOP-induced immunomodulation. Consistent with the suppression of humoral immunity and macrophage functions, the rats treated with $0.5 \times \mathrm{LD}_{50}$ DOP also manifested considerable increase in the lethal effects of endotoxin of $E$. coli indicating the likely impairment in endotoxin detoxification mechanism (glutathione-S-transferase) as reported with $2,3,7,8$, tetrachlorobenzo-p-dioxin. ${ }^{7)}$ Coincident with the enhanced endotoxin susceptibility seen in DOP-treated rats was an increase in parasite counts in the intestine of DOP-treated rats suggesting the failure on the part of the host to expel parasites consequent to impairment of immune functions. Since the mechanism of expulsion of $N$. brasiliensis has been suggested to involve immunological processes such as antibodies, sensitized T-lymphocytes and biogenic amines. ${ }^{11)}$ Recently some of the parasite models like Plasmodium and Trichinella have been examined in rodents for use as end points in immunotoxicity assessment of environmental chemicals. The present study adds to the list yet another good parasitic model of $N$. brasiliensis which manifests an increased worm burden in the presence of DOP.

In conclusion our data indicate that short term exposure to DOP brings about alteration in the functioning of rodent's immune system which may render the host more susceptible to endotoxin as well as impairs the rejection of $N$. brasiliensis parasite. Nevertheless studies are currently underway to further assess the alteration in host resistance and reversibility of phthalate-induced immunomodulation of host with other infection models.

\section{REFERENCES}

1) Thomas JA, Thomas JT. Biological effects of Di-(2-ethylhexyl) phthalate and other phthalic acid esters. CRC Crit Rev Toxicol 1984; 13: 283-318.

2) Dogra RKS, Khanna S, Nagale SL, Shukla LJ, Srivastava SN, Bhatnagar MC, Gupta PK, Shanker R. Effect of Di-octyl phthalate on immune system of rat. Indian J Exp Biol 1985; 23: 315-9.

3) Dean JH, Padarathsingh ML, Jerrells TR, Keys L, Northing JW. Assessment of immunobiological effects induced by chemicals, drugs or food additives. II: Studies with cyclophosphamide. Drug and Chem Toxicol 1979; 2: 133-53.

4) Saxena AK, Singh KP, Srivastava SN, Khanna S, Shukla LJ, Shanker R. Immunomodulating effects of caffeine $(1,3,7$,trimethylxanthine) in rodents. Indian J Exp Biol 1984; 22: 298-301.

5) Koller LD, Roan JC. Effects of lead and cadmium on mouse peritoneal macrophages. J Reticuloendo Soc 1977; 21: 7-12.

6) Katiyar JC, Gupta S, Sen AB. Action of diethylcarbamazine citrate on protective immunity in rats infected with Nippostrongylus brasiliensis. Z Parasitenkd 1985; 71: 
401-8.

7) Vos JG, Kreeftenberg JG, Engel HWB, Minderhond A, Van Noorle Jansen LM. Studies on 2,3,7,8 tetrachlorobenzo-p-dioxin induced immune suppression and decreased resistance to infection: Endotoxin hypersensitivity, serum zinc concentration and effect of thymosin treatment. Toxicol 1978; 9: 75-86.

8) Dogra RKS, Donaldson K, Khanna S, Shanker R. Lymphocytotoxicity of di-octyl phthalate (DOP) in rat. Presented in Symposium on Experimental and Molecular Pathology. IVRI, Izatnagar, India, 1984.

9) Gangolli SD. Testicular effects of phthalate esters. Environ Health Perspect 1982; 45: 77-84.

10) Fernandes G, Nair M, Onoe K, Tanaka T, Floyd R, Good RA. Impairment of cellmediated immunity function by dietary zinc deficiency in mice. Proc Natl Acad Sci 1979; 76: 457-61.

11) Ogilvie BM, Jones VE. Nippostrongylus brasiliensis: A review of immunity and the host parasite relationship in the rat. Exp Parasitol 1971; 29: 138-77.

Immunotoxicology Laboratory,

Industrial Toxicology Research Centre,

PB No. 80, MG Marg,

Lucknow-226001, India

*Division of Parasitology,

Central Drug Research Institute,

PB No. 173, Lucknow-226001, India.
Ram K.S. DOGRA

Santosh KHANNA

Lalji SHUKLA

Satyanarayan SRIVASTAVA

Suman GUPTA*

Jagdish C. KATIYAR* and

Ravi SHANKER

(Received November 20, 1986 and in revised form March 10, 1987) 